\title{
Improvement and Decline in Tactile Discrimination Behavior after Cortical Plasticity Induced by Passive Tactile Coactivation
}

\author{
Amra Hodzic, ${ }^{1,2}$ Ralf Veit, ${ }^{1}$ Ahmed A. Karim,,${ }^{1,2}$ Michael Erb, ${ }^{3}$ and Ben Godde ${ }^{1,4}$ \\ ${ }^{1}$ Institute of Medical Psychology and Behavioral Neurobiology, University of Tübingen, D-72074 Tübingen, Germany, ${ }^{2}$ International Max Planck Research \\ School, Graduate School of Neural and Behavioral Sciences, D-72074 Tübingen, Germany, ${ }^{3}$ Department of Neuroradiology, Section on Experimental \\ Magnetic Resonance of the Central Nervous System, D-72076 Tübingen, Germany, and ${ }^{4}$ Department of Neurology, University Hospital, D-72076 Tübingen, \\ Germany
}

Perceptual learning can be induced by passive tactile coactivation without attention or reinforcement. We used functional MRI (fMRI) and psychophysics to investigate in detail the specificity of this type of learning for different tactile discrimination tasks and the underlying cortical reorganization. We found that a few hours of Hebbian coactivation evoked a significant increase of primary (SI) and secondary (SII) somatosensory cortical areas representing the stimulated body parts. The amount of plastic changes was strongly correlated with improvement in spatial discrimination performance. However, in the same subjects, frequency discrimination was impaired after coactivation, indicating that even maladaptive processes can be induced by intense passive sensory stimulation.

Key words: functional MRI; psychophysics; perceptual learning; input statistics; somatosensory cortex; human

\section{Introduction}

The term "perceptual learning" describes lasting changes in the perception of stimuli after exercise of or experience with these stimuli (Gibson, 1963). Improvement in the extraction of specific stimulus parameters is highly specific for the learned stimulus parameters and can hardly be generalized. Therefore, it is suggested that this kind of perceptual learning is based mainly on plasticity in early processing stages (Fahle, 2002). Indeed, it has been shown that improved sensory and motor performance after extensive use and training is paralleled by a profound reorganization in primary sensory and motor cortices characterized by an enlargement of the representations of the trained body parts (Elbert et al., 1995; Pantev et al., 1998; Recanzone, 2000; Dinse and Merzenich, 2002).

Recent studies using electroencephalography (EEG) and magnetoencephalography (MEG) suggest that passive tactile coactivation without attention directed to the stimuli is able to induce similar plastic changes in the somatosensory cortex. Contrary to the view that learning requires top-down modulation by attention or reinforcement, these purely input-dependent changes also lead to perceptual improvement, the amount of which is predictable from the amount of reorganization (Godde et al.,

Received Aug. 11, 2003; revised Nov. 10, 2003; accepted Nov. 17, 2003

This work was supported by Volkswagen-Stiftung Grant 173035 (B.G.) and by a stipend from the Tübingen Max Planck Institute for Biological Cybernetics (A.H.). We thank Dr. Christoph Braun for technical support in the fMR measurements.

Correspondence should be addressed to Dr. Ben Godde, Institute of Medical Psychology and Behaviora Neurobiology, University of Tübingen, Gartenstrasse 29, D-72074 Tübingen, Germany. E-mail: benjamin.godde@uni-tuebingen.de.

D0I:10.1523/JNEUROSCI.3731-03.2004

Copyright $\odot 2004$ Society for Neuroscience $\quad 0270-6474 / 04 / 240442-05 \$ 15.00 / 0$
2000, 2003; Pleger et al., 2001). However, the cortical mechanisms of this type of passive learning, as well as principle differences and similarities with active learning processes, are not well understood, and it might be argued that improvement in discrimination performance is based on unspecific and transient sensitization caused by stronger cortical activation.

Here, we combined fMRI measurements with psychophysical tests of human spatial and frequency discrimination abilities to investigate in detail alterations in cortical topography and the task specificity of the described learning effects that were induced by the same stimulation protocol as in the above-mentioned EEG and MEG studies. This protocol was characterized by a few hours of tactile coactivation of small skin portions of the tip of the right index finger (IF) to evoke synchronous neural activity and to follow closely the idea of Hebbian learning [Dinse et al. (2003), their Fig. S1].

\section{Materials and Methods}

Eleven right-handed healthy subjects (average age, 29 years) participated in this study after giving informed consent. The work was conducted in strict accordance with the local ethics policies. The experiments consisted of a premapping, a coactivation procedure of $3 \mathrm{hr}$ duration, and a postmapping. fMRI mapping procedures were accompanied by psychophysical measurements of spatial and frequency discrimination performance.

fMRI mapping. Mapping of somatosensory cortical representations of the right (test) and left (control) index finger was done with a $1.5 \mathrm{~T}$ Magnetom Sonata (Siemens, Erlangen, Germany) whole-body MRI system equipped with a standard head coil. $\mathrm{T} 2{ }^{\star}$ weighted echoplanar images were acquired in axial orientation (repetition time, 3.0 sec; echo time, 60 msec; flip angle: $90^{\circ}$; matrix, $64 \times 64$; field of view, $192 \mathrm{~mm}$, 28 slices; slice thickness, $4 \mathrm{~mm}$; gap, $1 \mathrm{~mm}$; voxel dimension, $3 \times 3 \times 4 \mathrm{~mm}^{3}$ ) covering the whole brain. In addition, a T1-weighted three-dimensional 
data set containing 176 sagittal slices was obtained. Pneumatically driven tactile stimulation on the tip of the fingers was applied in a block design ( 192 volumes in 16 blocks, 12 volumes per block, each block of stimulation or rest lasting $36 \mathrm{sec}$ ). Four blocks of stimulation, each preceded by rest, were presented alternating on each finger. Stimulation blocks consisted of 108 stimuli applied with randomly varied interstimulus intervals between 250 and $400 \mathrm{msec}$, resulting in an average stimulation frequency of $3 \mathrm{~Hz}$. During rest, no stimulation was applied. To increase statistical power, this mapping procedure was repeated twice, resulting in a total of 594 volumes per session.

Image processing and statistical evaluation. Using BrainVoyager 2000 (Brain Innovation BV, Maastricht, The Netherlands), functional data were coregistered to a high-resolution anatomical data set and normalized to Talairach space. Each individual data set was corrected for movement artifacts (translation in $x$-, $y$-, and $z$-directions and rotation around all three axes), spatially smoothed with a $4 \mathrm{~mm}$ Gaussian kernel and high-pass (cutoff period, $288 \mathrm{sec}$ ) and low-pass (4 sec) filtered.

Using general linear model analysis, fMRI-activation compared with rest was evaluated by correlation with a boxcar function of alternating rest and stimulus conditions convolved with a canonical hemodynamic delay (delta value, $2.5 \mathrm{sec}$ ). To reduce the chance of type I errors caused by multiple comparisons, first cortex-based statistics were performed using a gray-matter-only mask generated from the high-resolution anatomical images; second, Bonferroni correction was applied.

For each individual subject, separately for contralateral primary (SI) and secondary (SII) somatosensory cortical area activations, the number of significantly activated voxels $(p<0.05)$ were counted as a measure of representational area, and Talairach coordinates of centers of gravity (CoG) of the activations were determined. SI and SII were anatomically defined as areas located on the anterior wall of the postcentral gyrus (SI) or within the upper bank of the lateral sulcus (parietal operculum, SII) (Affifi and Bergman, 1998; Francis et al., 2000).

In addition, fMRI activation maps during stimulation before and after coactivation were statistically compared for the test and the control finger in random-effects group analysis. For this purpose the individual contrast images (a linear combination of the parameter estimates for each condition) were entered into a second-level analysis.

One subject had to be excluded from analysis because no clear activation areas could be defined after coactivation.

Psychophysics. For testing spatial discrimination performance we used a grating orientation task (GOT) in a two-alternative forced-choice paradigm (for details, see Van Boven et al., 2000). Stimuli consisted of eight hemispherical plastic domes with gratings cut into their surfaces, resulting in parallel bars and grooves of equal width at each dome (JVP Domes; Stoelting Co., Wood Dale, IL). Widths of the bars and grooves varied between domes in the range $0.25-3.0 \mathrm{~mm}$. Gratings were manually applied by the experimenter perpendicularly to the surface of the skin of the right and left index finger tip with a duration of $\sim 1.5 \mathrm{sec}$ and $\sim 2 \mathrm{~mm}$ of skin displacement. Ridges and grooves were oriented randomly parallel or orthogonal to the axis of the finger tip. Each dome was applied 20 times. Subjects were blindfolded and were asked to report the orientation of the grating. Trials in which the subjects failed to respond immediately were rejected, as well as trials in which there were visible movements between skin and domes. The gratings discrimination threshold was defined as the level at which $75 \%$ of the responses were correct and was determined by interpolating between groove widths with $75 \%$ correct responses. Performance at this level is midway between chance and perfect performance and is a standard psychophysical criterion for threshold determination (van Boven and Johnson, 1994).

For temporal discrimination tests, vibrotactile stimuli were played back on microloudspeakers connected to a computer. The square-wave stimuli applied to the fingertip by the solenoid of the loudspeaker lasted $500 \mathrm{msec}$, and the interval between two stimuli was $200 \mathrm{msec}$. In each trial a reference frequency of $30 \mathrm{~Hz}$ was presented twice, followed by a test stimulus with frequencies between 29 and $39 \mathrm{~Hz}$. Subjects had to decide within $500 \mathrm{msec}$, in a two-alternative forced-choice manner, whether the frequencies of the test stimuli were higher or lower than that of the reference stimulus. In this frequency discrimination task (FDT), 10 different test frequencies were presented 10 times each in random order, and the number of correct responses for each test frequency was summed for additional analysis.

Each subject underwent a $4 \mathrm{~d}$ pretest period (one session per day for each task) to have stable baseline levels of discrimination performance, which was reached in most subjects after one or two sessions of initial learning. One subject was not able to discriminate any frequencies within the range of test frequencies and was excluded from FDT analysis. Two other subjects did not reach a stable level of performance before coactivation and were also excluded.

Coactivation. To apply coactivation during the $3 \mathrm{hr}$ stimulation period, a small solenoid with a diameter of $8 \mathrm{~mm}$ was mounted to the tip of the right index finger and was used to transmit the tactile stimuli of the coactivation protocol to the skin. The solenoid allowed simultaneous stimulation of the skin portions of the index finger under the solenoid, leading to coactivation of all receptive fields within this area; for an estimate of receptive field sizes of the human index finger, see VegaBermudez and Johnson (1999). According to these data, receptive fields within $8 \mathrm{~mm}$ of the tip of the index finger overlap partially or not at all. The position of the solenoid on the fingertip was chosen to enclose the skin locations that were used for testing discrimination performance and for tactile stimulation in $\mathrm{fMRI}$ recordings. The timing of the coactivation protocol was the same as in our previous studies (Godde et al., 2000, 2003). To prevent habituation during stimulation over several hours, stimuli were applied with interstimulus intervals between 8 and 1761 $\mathrm{msec}$ in random order, resulting in a mean stimulation frequency of 1.7 $\mathrm{Hz}$. The duration of each pulse was $10 \mathrm{msec}$.

Pulses were recorded on tape and were played back via portable tape recorders, allowing unrestrained mobility of the subjects during the coactivation period. Coactivation stimuli were applied at suprathreshold intensities. Subjects were instructed not to attend the stimulation. In fact, all subjects resumed their normal day of work.

\section{Results}

Using fMRI to map the cortical representations of the right (test) and left (control) index finger, group statistics revealed significant activations in the contralateral postcentral gyrus (SI) and in the contralateral upper bank of the lateral sulcus near the posterior pole of the insula (SII). This holds for the presessions as well as for the postsessions (Fig. 1). Additional activation foci were observed irregularly in the ipsilateral SII and in other cortical regions, such as the prefrontal and frontal areas. However, because they were not found in all subjects under all conditions, and specific session effects were not significant in the group statistics, quantitative analysis was restricted to SI and SII.

Relative to the presessions the numbers of activated voxels in the postsessions were reduced for the test as well as for the control finger both in SI and SII. However, the reduction in representational area in contralateral SI and SII was stronger for the control than for the test finger. This interaction led to an increased interhemispheric asymmetry between the representations of the right and left IF, indicating a relative overrepresentation of the test finger compared with the control finger after coactivation. Different levels in overall activity between sessions are a common finding in neuroimaging and might be caused by changes in arousal or physiological parameters such as heart rate or blood pressure. To rule out such unspecific session effects, which occur both for the test and the control finger, we used the activation in the control condition for normalization. For this purpose, for each individual subject we divided separately for SI and SII the number of voxels in the left hemisphere after stimulation of the test finger by the number of voxels in the right hemisphere after stimulation of the control finger. On average, after coactivation this normalized area of activation in the left hemisphere increased from 1.59 to 3.08 in SI and from 0.99 to 3.47 in SII (hand $\times$ session interaction effect: $F_{(1,38)}=4.54 ; p<0.05$; repeated-measures ANOVA with hand as fixed factor and session 
as repeated measure), indicating that $3 \mathrm{hr}$ of coactivation result in a twofold to threefold enlargement of the contralateral SI and SII representations of the stimulated finger relative to the representation of the control finger.

This enlargement was paralleled by a shift of the CoG of the representations in inferior and lateral direction along the postcentral gyrus in SI and in a medial and inferior direction in SII. The mean shift of the CoG was $5.4 \pm 0.7 \mathrm{~mm}$ in SI contralateral to the stimulated finger and $12.7 \pm 3.5$ $\mathrm{mm}$ in SII (mean \pm SEM; $p<0.01$ for both) (Fig. 2). On average, no shift could be observed for the CoG in the hemisphere contralateral to the control finger $(0.4 \pm$ 1.4 and $0.3 \pm 2.7 \mathrm{~mm}$ for SI and SII, respectively; $p>0.4$ ).

Cortical reorganization was accompanied by changes in tactile discrimination performance. In the GOT for all subjects we found a significant improvement in performance for the test but not for the control finger (Fig. 3a). Spatial thresholds on the right IF were reduced by $\sim 16 \%$, from $1.14 \pm 0.06$ to $0.96 \pm 0.07 \mathrm{~mm}$ (mean \pm SEM at pre and post sessions). Thresholds for the left control finger remained stable $(1.22 \pm 0.09$ and $1.25 \pm$ $0.08 \mathrm{~mm}$; pre and post, respectively). Repeated-measures ANOVA with hand as the fixed factor and session as the repeated measure revealed the significance of the hand $\times$ session interaction $\left(F_{(1,20)}=13.61 ; p<0.002\right)$. Within $24 \mathrm{hr}$ after coactivation, thresholds returned to their initial values (1.18 \pm 0.07 and $1.25 \pm 0.08 \mathrm{~mm}$, right and left index finger, respectively). The shift of the CoG in SI was strongly correlated to performance changes in this task. Subjects with the strongest shift of CoG and therefore the largest amount of reorganization were those with the most improvement in the GOT, whereas subjects with less reorganization showed less improvement $\left(r^{2}=0.83 ; p<0.001\right)$. Even changes in SII were less strong but significant positively correlated to improvement in the GOT $\left(r^{2}=0.51 ; p<0.05\right)$.

In the FDT we calculated probabilities of correct responses for all test frequencies independent for both hands (Fig. 3b). The mean difference threshold, derived from a fit to the averaged correct response probability at each test frequency, was between -2.8 and $+3.0 \mathrm{~Hz}$ for the right index finger (frequencies below and above $30 \mathrm{~Hz}$, respectively) and -4.5 and $+2.5 \mathrm{~Hz}$ for the left index finger. Difference thresholds for the right index finger increased to -4.8 and $+5.8 \mathrm{~Hz}$ (lower and higher frequencies, respectively) after coactivation, indicating a worsening in performance (Fig. $3 b$ ). Thresholds for the control finger remained constant $(-4.3 \mathrm{~Hz}$ and $+3.8 \mathrm{~Hz})$. Repeated-measures ANOVA with session as the repeated measure and hand and frequency as fixed factors revealed a significant hand $\times$ session effect $\left(F_{(1,152)}=\right.$ 4.813; $p=0.03$ ) but no interaction with frequency. These effects as well as the effects in the GOT were reversible within $24 \mathrm{hr}$.

Interestingly, the effect of coactivation on frequency discrimination was also correlated to the shift of the CoG in SI but not in SII (SI, $r^{2}=0.72, p<0.01$; SII, $\left.r^{2}=0.14, p=0.37\right)$. However, in contrast with the GOT, subjects with the strongest CoG shift in SI

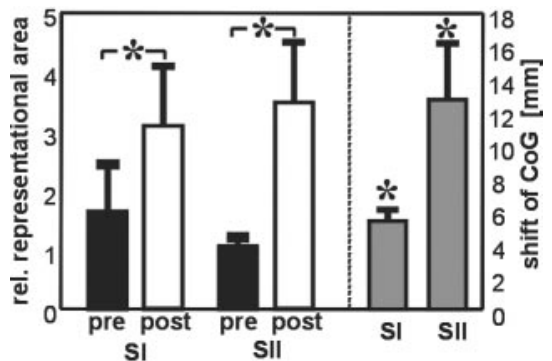

Figure 2. Changes in representational area and shift of $\mathrm{COG}$ (means $\pm S E M)$. On the left, the relative number of voxels (left vs right hemisphere) in SI and SII contralateral to the right IF before (pre, black columns) and after (post, white columns) coactivation are shown. In both areas, the relative size of the representation of the test finger is significantly increased. The parallel shift of the COG in SI and SII is shown on the right (gray bars). ${ }^{*} p<0.05$.

were those with little decline in frequency discrimination performance, whereas subjects with only a modest shift of CoG in SI showed the most worsening in the FDT. In addition, direct comparison of both behavioral tasks revealed that subjects with the most improvement in the GOT showed little decline in the FDT, whereas subjects with strongest impairment in the FDT were those with little improvement in the GOT $\left(r^{2}=0.61 ; p<0.05\right)$ (Fig. $4 c$ ).

\section{Discussion}

We found that after passive tactile coactivation, gratings orientation discrimination with the stimulated finger had improved. The quantity of this improvement was strongly correlated with the amount of reorganization in the contralateral SI cortex. Here- 
a
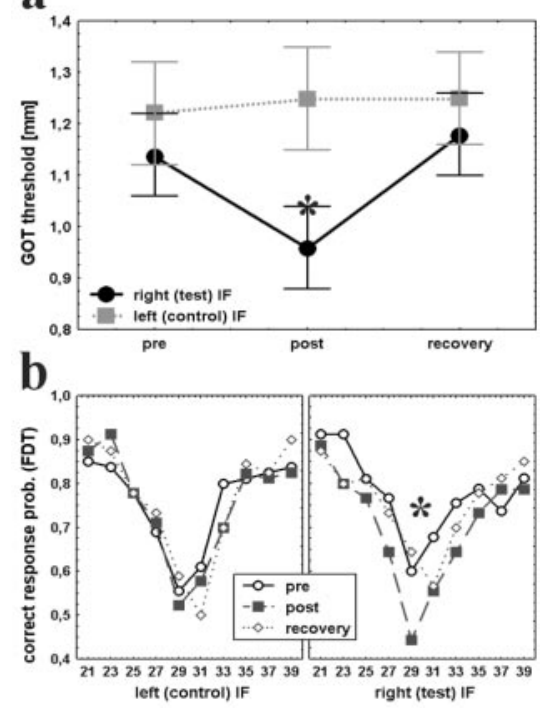

test frequency $[\mathrm{Hz}]$

Figure 3. Coactivation-induced changes in tactile discrimination performance. $a$, Thresholds in the GOT before (pre) and after (post) tactile coactivation on the right IF and after a $24 \mathrm{hr}$ recovery period (recovery) are shown for the control (squares) and the test (circles) finger (means \pm SEM). Reduction in thresholds indicates improved performance in the post condition for the test but not the control finger. $b$, Probability of correct responses in the FDT before (circles) and after (squares) coactivation and after recovery (diamonds) for the test (right) and the control (left) finger. Reduction in correct response probability for the test but not the control finger reveals a decline in frequency discrimination performance. ${ }^{*} p<0.05$.

with, our findings are in line with recent studies of Pleger et al. (2001) using EEG and our own experiments performed with MEG (Godde et al., 2003), which demonstrated a similar inferior and lateral shift of the N20 dipole in SI together with an improvement in spatial two-point discrimination performance. However, our results extend these findings in several aspects.

Surprisingly, the results from the FDT experiments were completely different from the GOT, laying open the specificity of coactivation-induced perceptual learning. In addition, our data reveal that reorganization induced by passive tactile coactivation is also evident in SII. As well as for SI, the contralateral SII representation of the right index finger was enlarged and the amount of reorganization was correlated with the improvement in spatial discrimination performance. However, there was no relationship between changes in SII and decline in frequency discrimination.

This is in good agreement with earlier studies in monkeys (Mountcastle et al., 1969, 1990; Recanzone et al., 1992) and humans (Francis et al., 2000; Harrington et al., 2001), showing the predominant role of SI in processing and discrimination training of flutter stimuli.

Furthermore, topographic organization in SII is not as pronounced as in SI, which might explain the lower correlation strength between the CoG shift in SII and the improvement in the GOT performance.

In the classical view, feedback and attention are crucial for learning processes. Therefore, it might be surprising that human spatial discrimination performance is subject to improvement within a few hours of passive tactile coactivation without invoking training, attention, or reinforcement, and one might argue that behavioral changes are caused by an unspecific rise in cortical activity or an increase of sensitivity. However, this argument fails to explain the decline in frequency discrimination performance. Different and opposite effects on the performance in spatial and

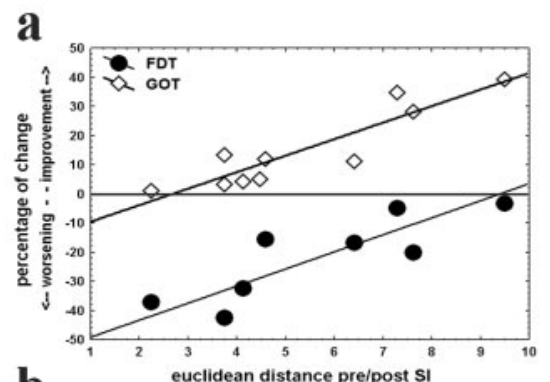

b
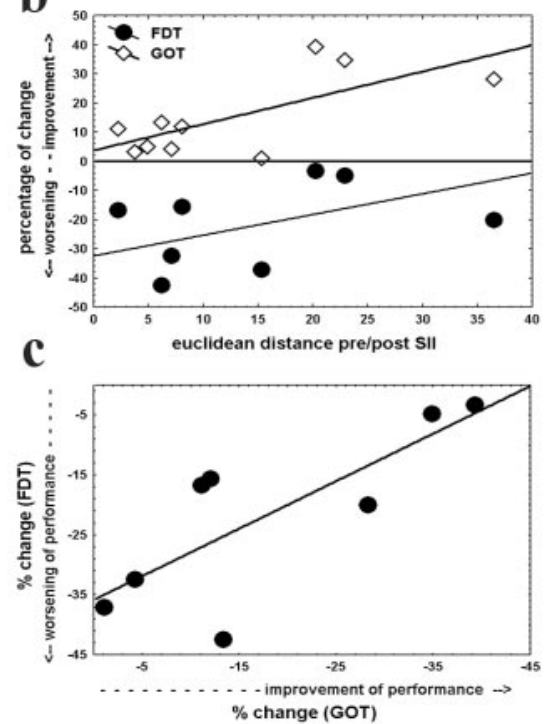

Figure 4. Relationship between cortical reorganization and alterations in discrimination performance. Changes in GOT thresholds (diamonds) and the reduction in correct response probability in the FDT (circles) are plotted against the shift of the CoG in SI ( $a$ ) and SII (b). To simplify direct comparison between GOT and FDT performance, absolute values of spatial threshold changes are used. Therefore, for both tasks, positive values indicate an improvement in performance and negative values a decline. Reorganization in $\mathrm{SI}$ is strongly correlated with performance changes in both tasks (GOT, $r^{2}=0.83, p<0.001$; FDT, $r^{2}=0.72, p<0.01$ ), whereas for SII, significant correlation was found only for the GOT $\left(r^{2}=0.51 ; p<0.05 ; r^{2}=\right.$ $0.14 ; p=0.37)$. $c$, Alterations in correct response probability (FDT) are plotted against changes in spatial thresholds (GOT), revealing that a stronger improvement in the GOT is correlated to less but an existent decline in the FDT $\left(r^{2}=0.61 ; p<0.05\right)$.

temporal discrimination tasks together with the high correlation between perceptual and cortical changes indicate that the plastic processes in the primary and secondary somatosensory cortex are scaled to the degree of perceptual improvement and decline in a highly specific manner. Even for the visual system it has been shown that perceptual learning occurs without conscious perception of repetitive applied subthreshold stimuli (Watanabe et al., 2001).

A recent pharmacological study revealed the importance of NMDA-dependent receptors for this type of cortical plasticity and learning (Dinse et al., 2003). Together with the findings that no effect of tactile single-point stimulation on cortical organization and discrimination behavior can be observed (Dinse et al., 2002) and that precise timing of coactivation is crucial for the induction of plastic changes (Van der Berg and Dinse, 2002), it is likely that the described learning effects are based on associative, and therefore Hebbian-type, cortical mechanisms.

The effectiveness of the passive coactivation protocol might be explained by the intensity of the stimulation. Selected skin regions were stimulated with $1.7 \mathrm{~Hz}$ for $3 \mathrm{hr}$. This resulted in $\sim 10,000$ single stimuli, which is much more than in most active training and perceptual learning experiments. The improvement 
in the GOT can be explained by the growing number of sensory cortical neurons involved in tactile processing resulting from this intensive stimulation. However, this cannot clarify the worsening in the FDT; moreover, it is contradictory.

One possible explanation is based on the important role of the adaptive capacity of the brain to generate the most behaviorally useful representation of the individual sensory environment. Competition between representations is one of the fundamental principles of cortical plasticity. Assuming a dynamic steady state of the neural system in which (based on competition) the processing of many different tasks is optimized for overall performance of the whole system but not for optimal processing of a specific task, unbalancing of the system by extensive learning of one task might result in decline in another one. The tactile stimuli in our coactivation paradigm were applied with a low average frequency of $1.7 \mathrm{~Hz}$. Therefore, based on competition, the steady state of cortical processing might be biased to superior processing of pressure stimuli on the cost of stimuli in the flutter range. Competition is strongest when few additional neurons are recruited, as indicated by only a small shift of the CoG. In this condition, decline in the FDT performance should be maximal, as was the case. With the growing number of newly gained processing units the competition decreases and FDT performance can profit from this increase of representational area.

An additional explanation is supported by animal experiments. In rats, coactivation resulted not only in an increase of receptive field size and representational area but also in prolonged response durations of the neuronal populations (Godde et al., 1996). Assuming that tactile coactivation induces comparable changes in rats and humans, as shown for cortical reorganization, temporal neuronal response characteristics might be altered in humans as well, resulting in less sharp temporal response profiles. As a result, temporal discrimination might be impaired. Our correlation analysis revealed that subjects with little cortical reorganization showed the most worsening in frequency discrimination, whereas subjects with large shifts of the CoG in SI, were little impaired after coactivation, indicating that ameliorating effects of prolonged response duration on frequency discrimination might be compensated by an increased number of processing units.

The perspective to induce cortical reorganization and perceptual learning with purely passive tactile stimulation sounds promising even for rehabilitation after cortical lesions or deafferentation, and first attempts to treat patients suffering from phantom limb pain with passive tactile coactivation have been successful (Huse et al., 2001). However, even maladaptive phenomena such as focal dystonia, tinnitus, as well as phantom limb pain, are often associated with similar plastic cortical reorganization as described previously (Flor et al., 1995; Elbert et al., 1998; Muhlnickel et al., 1998; Lotze et al., 1999), and the present study reveals for the first time that even a decline in behavioral performance can be induced in healthy subjects by this type of coactivation. Therefore, we suggest that the consequences of this input-dependent massive and short-term alterations of cortical processing should be carefully investigated and compared with active training and learning processes developing over longer time scales to prevent maladaptive effects of such learning without effort.

\section{References}

Affifi AK, Bergman RA (1998) Functional neuroanatomy, text and atlas. New York: McGraw-Hill.

Dinse HR, Merzenich MM (2002) Adaptation of inputs in the somatosen- sory system. In: Perceptual learning (Fahle M, Poggio T, eds), pp 19-42. Boston: MIT.

Dinse HR, Kreikemeier K, Van der Berg I, Böhmer G, Wiemer J (2002) Manipulating input correlations: complementary forms of cortical map reorganization and perceptual learning. Soc Neurosci Abstr 28:840.3.

Dinse HR, Ragert P, Pleger B, Schwenkreis P, Tegenthoff M (2003) Pharmacological modulation of perceptual learning and associated cortical reorganization. Science 301:91.

Elbert T, Pantev C, Wienbruch C, Rockstroh B, Taub E (1995) Increased cortical representation of the fingers of the left hand in string players. Science 270:305-307.

Elbert T, Candia V, Altenmüller E, Rau H, Sterr A, Rockstroh B, Pantev C, Taub E (1998) Alteration of digital representations in somatosensory cortex in focal hand dystonia. NeuroReport 9:3571-3575.

Fahle M (2002) Introduction. In: Perceptual learning (Fahle M, Poggio T, eds), pp ix-xx. Cambridge, MA: MIT.

Flor H, Elbert T, Knecht S, Wienbruch C, Pantev C, Birbaumer N, Larbig W, Taub E (1995) Phantom-limb pain as a perceptual correlate of cortical reorganization following arm amputation. Nature 375:482-484.

Francis ST, Kelly EF, Bowtell R, Dunseath WJ, Folger SE, McGlone F (2000) fMRI of the responses to vibratory stimulation of digit tips. NeuroImage 11:188-202.

Gibson EJ (1963) Perceptual learning. Annu Rev Psychol 14:29-56.

Godde B, Spengler F, Dinse HR (1996) Associative pairing of tactile stimulation induces somatosensory cortical reorganization in rats and humans. NeuroReport 8:281-285.

Godde B, Stauffenberg B, Spengler F, Dinse HR (2000) Tactile coactivationinduced changes in spatial discrimination performance. J Neurosci 20:1597-1604.

Godde B, Ehrhardt J, Braun C (2003) Behavioral significance of inputdependent plasticity of human somatosensory cortex. NeuroReport 14:543-546.

Harrington GS, Hunter Downs III J (2001) FMRI mapping of the somatosensory cortex with vibratory stimuli: is there a dependency on stimulus frequency? Brain Res 897:188-192.

Huse E, Preissl H, Larbig W, Birbaumer N (2001) Phantom limb pain. Lancet 358:1015.

Lotze M, Grodd W, Birbaumer N, Erb M, Huse E, Flor H (1999) Does use of a myoelectric prosthesis prevent cortical reorganization and phantom limb pain? Nat Neurosci 2:501-502.

Mountcastle VB, Steinmetz MA, Romo R (1990) Frequency discrimination in the sense of flutter: psychophysical measurements correlated with postcentral events in behaving monkeys. J Neurosci 10:3032-3044.

Mountcastle VB, Talbot WH, Sakata H, Hyvärinen J (1969) Cortical neuronal mechanisms in flutter-vibration studied in unanaesthetized monkeys: neuronal periodicity and frequency discrimination. J Neurophysiol 32:452-484.

Muhlnickel W, Elbert T, Taub E, Flor H (1998) Reorganization of auditory cortex in tinnitus. Proc Natl Acad Sci USA 95:10340-10343.

Pantev C, Oostenveld R, Engelien A, Ross B, Roberts LE, Hoke M (1998) Increased auditory cortical representation in musicians. Nature 392:811-814.

Pleger B, Dinse HR, Ragert P, Schwenkreis P, Malin JP, Tegenthoff M (2001) Shifts in cortical representations predict human discrimination improvement. Proc Natl Acad Sci USA 98:12255-12260.

Recanzone GH (2000) Cerebral cortex plasticity: perception and skill acquisition. In: The new cognitive neurosciences (Gazzaniga MS, ed), pp $237-$ 250. Boston: MIT.

Recanzone GH, Merzenich MM, Jenkins WM, Grajski KA, Dinse HR (1992) Topographic reorganization of the hand representation in cortical area $3 \mathrm{~b}$ of owl monkeys trained in a frequency-discrimination task. J Neurophysiol 67:1031-1056.

Van Boven RW, Johnson KO (1994) A psychophysical study of the mechanisms of sensory recovery following nerve injury in humans. Brain 117:149-167.

Van Boven RW, Hamilton RH, Kauffmann T, Keenan JP, Pascual-Leone A (2000) Tactile spatial resolution in blind Braille readers. Neurology 54:2230-2236.

Van der Berg I, Dinse HR (2002) What is "simultaneous"? Tactile coactivation in human subjects reveals requirement for millisecond precision for induction of plastic changes. Soc Neurosci Abstr 28:650.17.

Vega-Bermudez F, Johnson KO (1999) SAl and RA receptive fields, response variability, and population responses mapped with a probe array. J Neurophysiol 81:2701-2710.

Watanabe T, Nanez JE, Sasaki Y (2001) Perceptual learning without perception. Nature 413:844-848. 